INPLASY

PROTOCOL

To cite: Xu et al. The clinical efficacy of traditional Chinese medicine in the treatment of viper bites A meta-analysis. Inplasy protocol 202190050. doi:

10.37766/inplasy2021.9.0050

Received: 16 September 2021

Published: 16 September 2021

Corresponding author:

Xiaogang $\mathrm{Xu}$

1909715363@qq.com

Author Affiliation:

Jiangxi University of Chinese Medicine.

Support: NSFC.

Review Stage at time of this submission: Data extraction.

Conflicts of interest:

None declared.

\section{The clinical efficacy of traditional Chinese medicine in the treatment of viper bites A meta-analysis}

Xu, XG1; Wang, Y2; Xu, YF3; Dong, X4; Zhang, DL5

Review question / Objective: To apply meta-analysis to comprehensively evaluate the clinical efficacy of traditional Chinese medicine in the treatment of viper bites.

Condition being studied: According to literature surveys, relevant departments of many hospitals across the country have used in-hospital Chinese medicine preparations in combination with conventional methods to treat the disease, which have played a certain role in improving clinical efficacy, alleviating local symptoms, reducing complications and shortening the course of the disease.

INPLASY registration number: This protocol was registered with the International Platform of Registered Systematic Review and Meta-Analysis Protocols (INPLASY) on 16 September 2021 and was last updated on 16 September 2021 (registration number INPLASY202190050).

\section{INTRODUCTION}

Review question / Objective: To apply meta-analysis to comprehensively evaluate the clinical efficacy of traditional Chinese medicine in the treatment of viper bites.

Condition being studied: According to literature surveys, relevant departments of many hospitals across the country have used in-hospital Chinese medicine preparations in combination with conventional methods to treat the disease, which have played a certain role in improving clinical efficacy, alleviating local symptoms, reducing complications and shortening the course of the disease. 


\section{METHODS}

Search strategy: \#1"snakebite" [MeSH] OR "snake bite" [MeSH] OR " snakebites "[Title/Abstract] OR " bite, snake " [Title/Abstract] OR "envenomation, snakebite "[Title/Abstract] OR " snakebite envenomation " [Title/ Abstract] OR " snakebite envenomations " [Title/Abstract] | \#2"traditional Chinese medicine "[MeSH] OR " traditional medicine, Chinese " [Title/Abstract] OR " Chinese traditional medicine "[Title/ Abstract] OR " Chinese medicine, traditional " [Title/Abstract] | \#3 \#1AND\#2 | \# 4 "randomized controlled trial" [MeSH] OR " clinical trials, randomized" [MeSH] OR " trials, randomized clinical" [Text Word] OR " controlled clinical trials, randomized" [Text Word] OR " double blind* " [Text Word] OR " random allocat* " [Text Word] OR "double-blind method" [Text Word] | \#5 animal [MeSH] NOT human [MeSH] |\#6 \#4 NOT\#5 | \#7 \#3 AND\#6.

Participant or population: Patients, viper bite patients who meet international or domestic industry-recognized diagnostic standards are not limited by gender, age, ethnicity, source of cases, and disease severity.

Intervention: The experimental group received traditional Chinese medicine combined with conventional treatment, and the control group received only conventional treatment.

Comparator: Total effective rate, local symptom score, systemic symptom score, swelling score, pain score.

Study designs to be included: The study inclusion criteria were as follows: study design, randomized controlled trials (RCTs), the language is Chinese or English, and there is no limit to whether blinding is implemented; patients, viper bite patients who meet international or domestic industry-recognized diagnostic standards are not limited by gender, age, ethnicity, source of cases, and disease severity.

Eligibility criteria: study design, randomized controlled trials (RCTs), the language is Chinese or English, and there is no limit to whether blinding is implemented; patients, viper bite patients who meet international or domestic industry-recognized diagnostic standards are not limited by gender, age, ethnicity, source of cases, and disease severity.

Information sources: Computer searched PubMed, Cochrane library, CNKI, VIP database, Wanfang database and Chinese Biomedical Literature database to collect RCTs literature on the treatment of viper bites with traditional Chinese medicine.

Main outcome(s): In accordance with the search strategy, using computer search methods, a total of 460 related documents were obtained. After using Endnote software to check duplicates, 97 duplicate documents were excluded. By reading the titles and abstracts of the remaining documents, 340 documents were excluded according to the inclusion and ranking criteria.

Quality assessment / Risk of bias analysis: The included RCT studies were evaluated using the Cochrane Risk of Bias assessment tool.Using Stata 15.1 software to test the publication bias of the studies that recorded the total effective rate index.

Strategy of data synthesis: The RevMan5.4 software provided by the Cochrane Collaboration was used for meta-analysis of the included literature. For continuous variables, the mean difference (MD) is selected, and the relative risk (RR) is selected as the effect size analysis for binary variables, and the $95 \%$ confidence interval $(\mathrm{Cl})$ is calculated at the same time. Heterogeneity analysis between the results of the included studies was evaluated by using the chi-square test. If $12 \leq 50 \%, P \geq 0.1$, it indicates that there is homogeneity 
among multiple studies; if $12>50 \%, P<0.1$, it indicates If there is heterogeneity among multiple studies, the reasons for the heterogeneity shall be further analyzed, and the subgroup analysis shall be carried out according to related factors. In view of the clinical heterogeneity of interventions such as hospital preparations, the data in this study are analyzed by random effects models. If the heterogeneity between subgroups is too large, a descriptive analysis will be performed, and sensitivity analysis will be used to test the stability of the results.

Subgroup analysis: According to the treatment method of the experimental group, some studies were subgrouped, of which were hospital preparation group, and some studies were a combination of hospital preparations and Chinese patent medicines.

Sensitivity analysis: Using Stata 15.1 to conduct sensitivity analysis on the studies that recorded the total effective rate index, and in RevMan5.4 software, the effect size and $P$ value change were observed by excluding the included studies one by one.

Country(ies) involved: China.

Keywords: Traditional Chinese medicine; Agkistrodon halys bite; Meta-analysis.

Contributions of each author:

Author 1 - Xiaogang Xu.

Author 2 - Yu Wang.

Author 3 - Yifeng Xu.

Author 4 - Xin Dong.

Author 5 - Delin Zhang. 